\title{
THORACIC SPINE TRANSLOCATION WITHOUT CORD INJURY
}

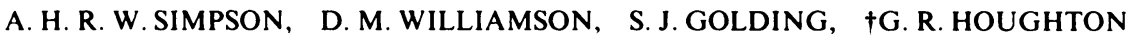

From Nuffield Orthopaedic Centre, Oxford

\begin{abstract}
We report three cases of thoracic spine translocation without neurological deficit. In each case bilateral pedicular fractures, demonstrated by computed tomography, produced 'floating arches' which account for the sparing of the cord. If computed tomography demonstrates adequate canal dimensions, these patients may be treated conservatively, but the treatment of choice at specialist spinal centres is operative stabilisation.
\end{abstract}

Unstable spinal fractures are frequently associated with cord injury. This is especially so in the thoracic spine where there is the least space around the neural elements (Denis 1983). Considerable violence is necessary to produce a fracture or dislocation of the upper thoracic spine; the narrow spinal canal in this region makes transection of the cord almost inevitable. Bohlman in 1985 and Holdsworth in 1970 stated that shearing fractures of the thoracic region are always associated with complete paraplegia.

We have recently seen three patients with severe thoracic translocation, but no neurological deficit. The anatomy of these injuries was studied with computed tomography (CT), which is the investigation of choice for assessing stability (Lynch, McManus and Ennis 1986); this explained the neural sparing. We discuss the specific problems of the diagnosis and management of this infrequent but important injury.

\section{CASE REPORTS}

Case 1. A 17-year-old motorcyclist was involved in a road traffic accident and sustained a minor head injury with brief loss of consciousness, and a fractured clavicle, humerus and ribs on the left side. Clinical examination of the spine showed no kyphus nor any other deformity of the spinous processes; neurological examination was normal. Radiographs showed a fracture dislocation at

A. H. R. W. Simpson, MA, FRCS, Orthopaedic Registrar

D. M. Williamson, MA, FRCS, Senior Orthopaedic Registrar

S. J. Golding, MA, FRCR, Director, Oxford Regional Computed Tomography Unit

+G. R. Houghton, deceased, MA, FRCS, Orthopaedic Consultant

Nuffield Orthopaedic Centre, Headington, Oxford OX3 7LD, England.

Correspondence should be sent to Mr A. H. R. W. Simpson.

(C) 1989 British Editorial Society of Bone and Joint Surgery

$0301-620 \mathrm{X} / 90 / 1192 \$ 2.00$

J Bone Joint Surg [Br] 1990; 72-B: 80-3.
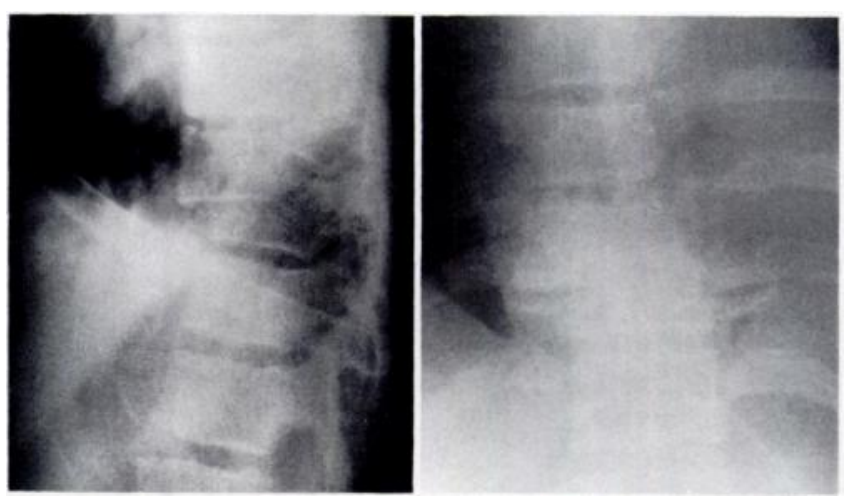

Fig. 1

Case 1. Radiographs showing fracture-dislocation of T9 upon T10.

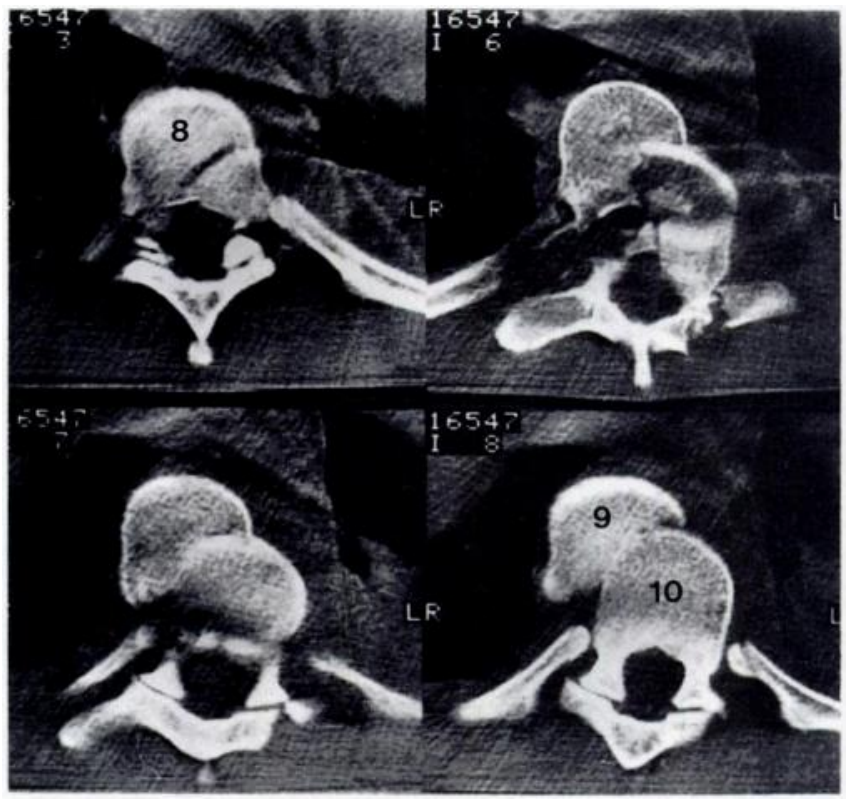

Fig. 2

Case 1. Axial CT images from T8 to T10, showing an unstable fracture on the left side of T8, separation of the body of T9 from its arch, and the body of T9 lying anterolateral to the body of T10. 
$\mathrm{T} 9 / 10$, with severe malalignment, the body of $\mathrm{T} 9$ being displaced anteriorly and to the right of the body of T10 (Fig. 1). CT confirmed the fracture dislocation, showing considerable separation of the body of T9 from its neural arch (Fig. 2). There was also a left pleural collection, consistent with a haemothorax. CT also demonstrated an unsuspected and unstable fracture at T8, with a transverse fracture of the pedicle and transverse process on the left, and an isolated fracture of the base of the right pedicle of $\mathrm{T} 7$. Ten days after injury the spine was reduced by Harrington instrumentation and the patient then mobilised in a jacket.

The rods were removed after one year at which time the patient was asymptomatic. Follow-up CT at 15 months after treatment showed healing of the fractures with no significant stenosis at any level. The smallest anteroposterior diameter of the spinal canal was $14.7 \mathrm{~mm}$ (normal > $11.5 \mathrm{~mm}$, Ullrich et al (1980).

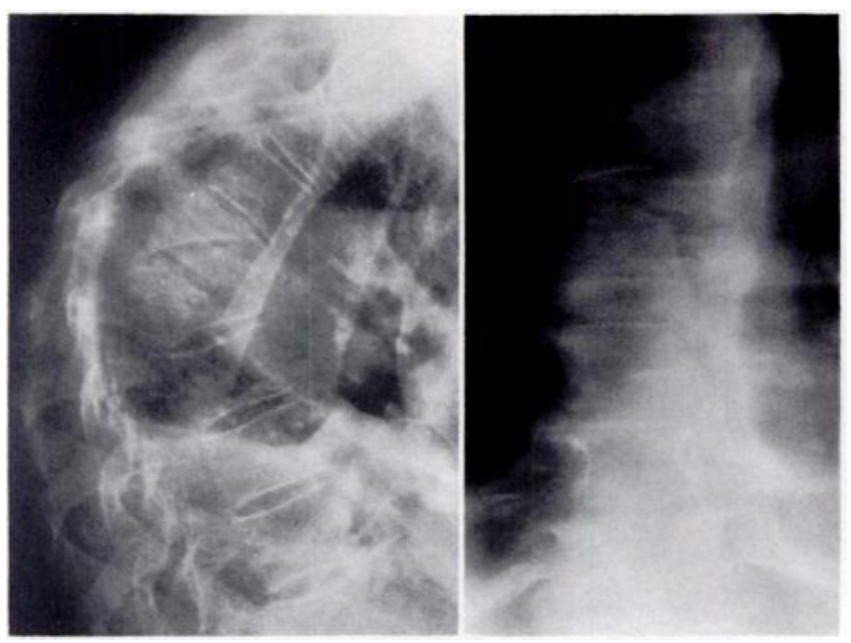

Fig. 3

Case 2. Lateral radiograph and AP tomogram showing angular kyphosis between $\mathrm{T} 5$ and $\mathrm{T} 7$, with severe compression and displacement of the T6 vertebral body.

Case 2. A 54-year-old lady was thrown off her horse, landing on the back of her left shoulder. A casualty officer diagnosed fractures of the left third and fourth ribs, both clinically and radiologically, and noted that there was no spinal tenderness.

Seven weeks later a further radiograph was taken because of persisting left chest pain; this showed malalignment of the thoracic spine and a left pleural collection. The fracture was difficult to see because of the dense overlying soft tissue shadows of the heart and aorta and the full extent of the injury was still not apparent. However, radiographs and anteroposterior tomograms of the thoracic spine showed severe lateral displacement at T7 with an angular kyphosis (Fig. 3). Clinical examination at this stage showed only minimal local tenderness and altered sensation in the distribution of the seventh thoracic nerve, but no long tract signs or sphincter disturbance.

CT confirmed the fracture-dislocation of $\mathrm{T} 6 / 7$, showing an oblique comminuted fracture through several levels, with displacement of the upper fragments to the left of the lower ones (Fig. 4). In view of the minimal symptoms and the delay in diagnosis, conservative management was continued. By 15 weeks from the time of injury there was full clinical recovery apart from persistence of an asymptomatic thoracic kyphosis.

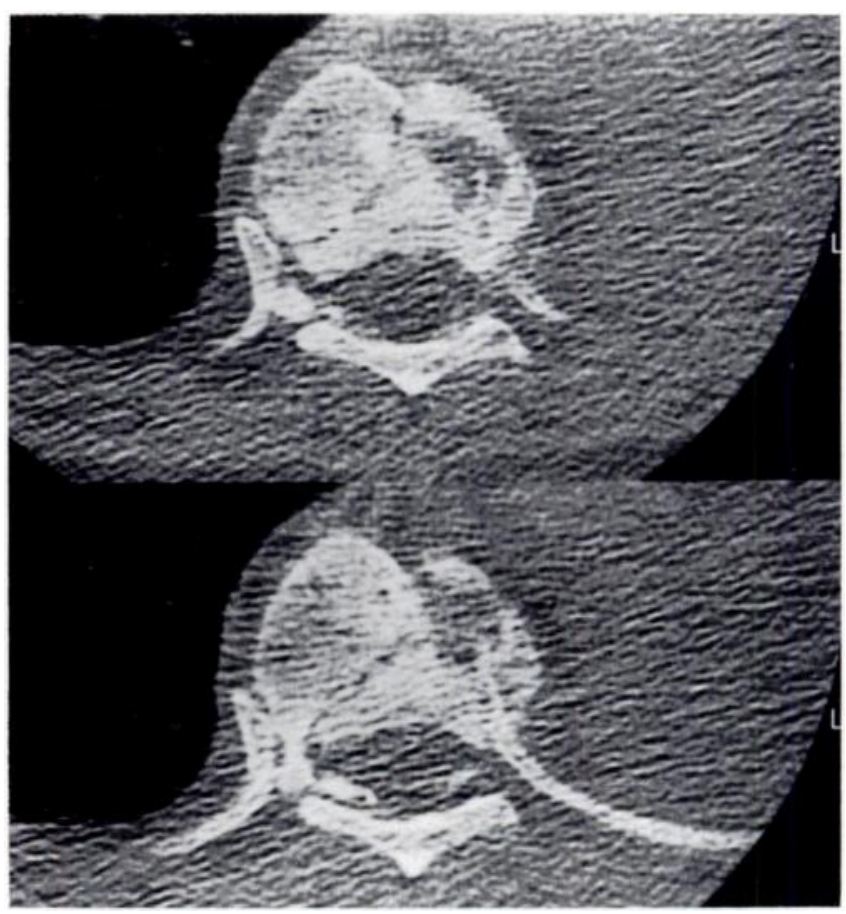

Fig. 4

Case 2. Axial CT sections showing the vertebral bodies lying side by side, with preservation of the spinal canal.

Case 3. A 23-year-old woman was involved in a motorcycle accident. She sustained a moderate head injury, an open comminuted fracture of the left tibia associated with arterial occlusion requiring a vein graft, and open fractures of the right distal femur and proximal tibia. There were also fractures of the left humerus and olecranon. After five days, she was transferred to this centre with a gangrenous left leg which required belowknee amputation. The wounds on the right leg had been closed primarily and were now grossly infected.

At this stage, her spinal injury was recognised (Fig. 5). CT showed a severely comminuted fracture of the body of T7, with marked shortening of the spine, the superior fragments passing to the right of the inferior (Fig. 6). Although the vertebral body had fractured from 
the pedicles, the arch was intact and articulated normally with the adjacent levels, so that the shortening had produced an acute kyphosis. There was also an oblique fracture through the $T 7 / 8$ facet joint and a right haemothorax.

In view of the patient's general debility the spinal injury was managed conservatively. Tomograms at six weeks after the injury showed that the spinal fractures were uniting. At review two years after injury the patient had no symptoms from her spine, nor any of spinal stenosis.

\section{DISCUSSION}

This major spinal injury can be surprisingly difficult to diagnose both clinically and radiologically: the diagnosis was initially missed in two of our three cases. Clinical examination often fails to reveal any step or gibbus because the posterior elements, in particular the spinous processes and supraspinous ligaments, have remained in alignment. Spinal tenderness may be absent. A chest radiograph, performed primarily for the soft tissues, does not show this region of the spine well. However, Dorr, Harvey and Nickel (1982) reported a $36 \%$ incidence of haemothorax in patients with thoracic rotation and shear fractures, so the presence of a haemothorax is an important warning sign.

There is debate on the stability of acute spinal translocations in the thoracic region. Holdsworth (1970) considered that a shearing fracture was a stable injury, but Sasson and Mozes (1987) and Bohlman (1985) deem it to be highly unstable. The rib cage (Dorr et al 1982) and the strong costovertebral ligaments confer significant stability to the thoracic vertebral fractures and dislocations, and it is possible that the variable damage to these elements explains the divergence of viewpoints on stability. If there has been significant damage to a number of ribs in the region of the translocation, then the injury is likely to be unstable. We consider that all such injuries should be regarded as unstable.

Many patients with spinal injuries have suffered multiple trauma and are often unconscious on arrival in the emergency reception area. If plain radiographs suggest that there has been complete cord transection and that any paraplegia is irrevocable, it may be considered that no further cord damage is possible and that there is no need to institute the handling routine for an unstable spine. Our cases demonstrate that plain radiographs should never be used to infer the state of the spinal cord.

Complete dislocation of the cervical spine and major fracture-dislocation of the lumbar region with neurological sparing have been reported (Jacobs 1977; Pitman, Pitman and Greenberg 1977), but the neurological sparing after severe displacement in the thoracic region is more remarkable because of the less favourable dimensions of the cord and the canal in this region. We
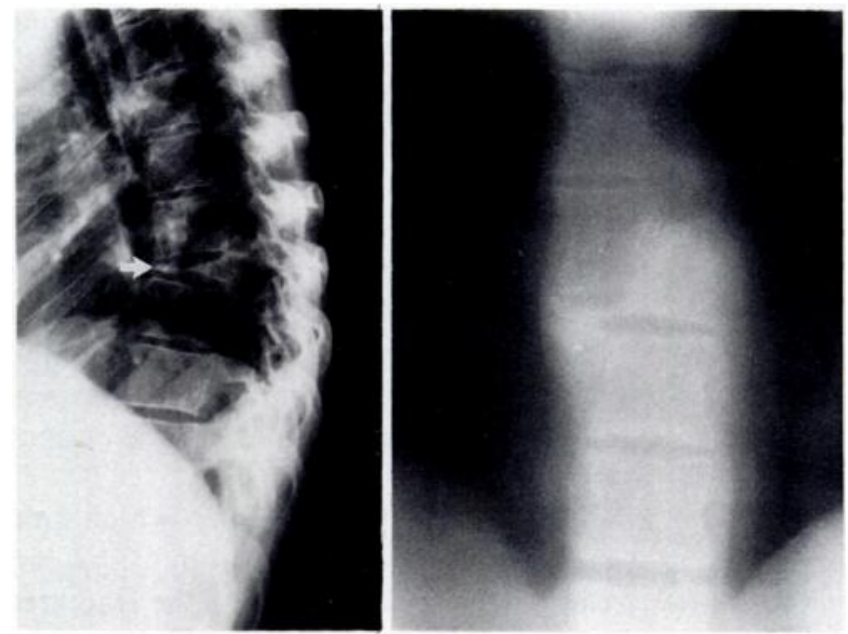

Fig. 5

Case 3. Lateral radiograph showing severe compression of the body of T7 (arrow), and AP tomogram showing separation of the fragments of the body of $\mathrm{T} 7$, with the $\mathrm{T} 6 / 7$ and $\mathrm{T} 7 / 8$ discs almost level.

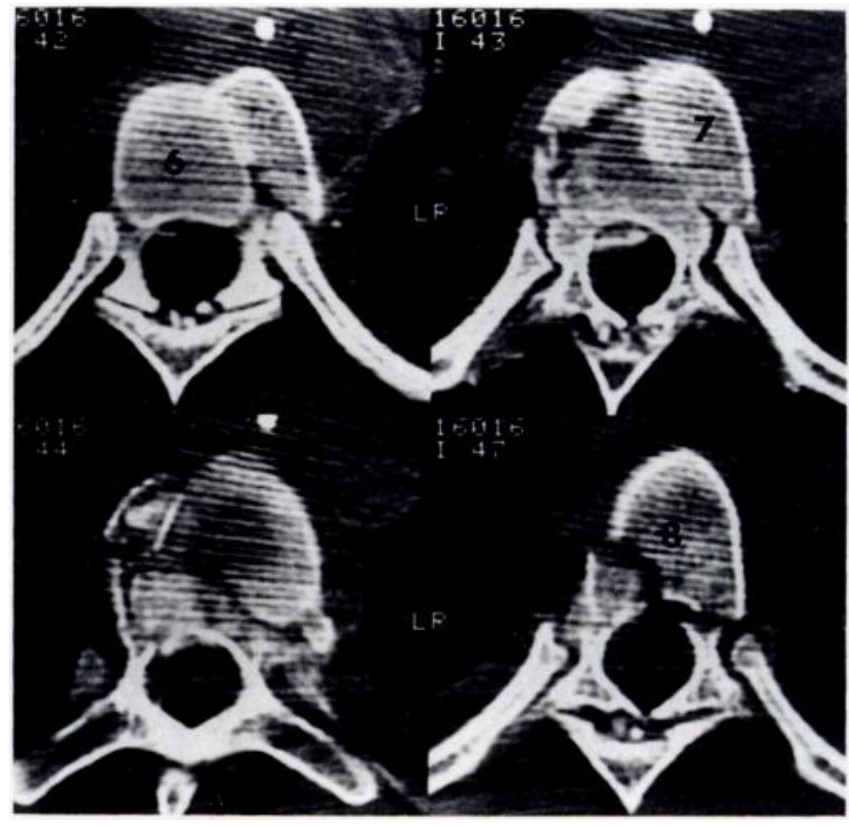

Fig. 6

Case 3. Series of CT sections showing the fragments of the body of T7 lying around those of $\mathrm{T} 6$, and an unstable fracture at T8. Note that the posterior elements remain in line.

could find only two reports of similar injuries (Gertzbein and Offierski 1979; Sasson and Mozes 1987), but in neither of these was $\mathrm{CT}$ available to delineate the fracture pattern and canal dimensions accurately.

Since its introduction, CT has become established as the investigation of choice for patients with suspected spinal fracture. It gives a precise and comprehensive delineation of the fracture, so that the degree of spinal stenosis and stability can be predicted accurately (Faerber 


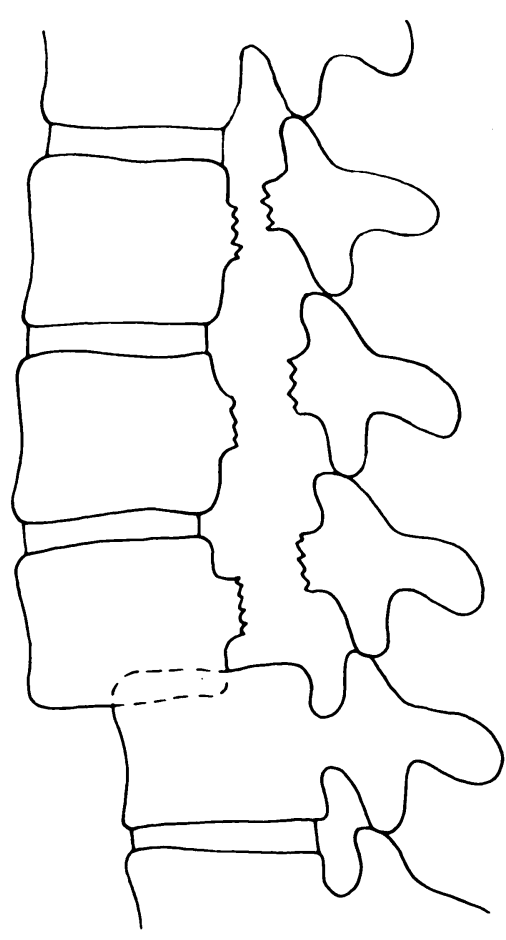

Fig. 7

Diagram showing how the 'floating arches' remain in line.

et al 1979; Lynch et al 1986). In each of our cases CT demonstrated a pattern of injury which explained the cord sparing. In essence, bilateral pedicular fractures at several levels had allowed the posterior elements to remain in continuity whilst the vertebral bodies became severely displaced (Fig. 7). The cord had remained in its correct relationship to the posterior elements and was therefore not compromised.

Hunter et al (1987) suggest that complex malalignment involving displacement and rotation of fragments is best appreciated on three-dimensional CT reconstructions and suggest that this technique may also reveal fractures of the posterior elements which are not seen on two-dimensional images. In our experience, however, reformatted three-dimensional views have failed to yield any further diagnostic information.

For the two cases reported previously (Gertzbein and Offierski 1979; Sasson and Mozes 1987) opposite conclusions were reached as regards the need for operative stabilisation. In our series, the systemic sepsis in case 3 precluded internal fixation, CT had shown adequate canal dimensions and a satisfactory outcome was obtained by bed rest and 'log-rolling' until union. In case 2 the patient had already been mobilised with the spinal fracture and, although at present asymptomatic, has a severe kyphosis. In case 1 operative stabilisation resulted in an excellent outcome and allowed early mobilisation.

Conclusions. If computed tomography demonstrates adequate dimensions throughout the spinal canal a good result can be obtained by skilled conservative management, but if the necessary expertise is available and the patient's condition permits, operative stabilisation may be the treatment of choice. This should, of course, be attempted only at specialist spinal centres.

No benefits in any form have been received or will be received from a commercial party related directly or indirectly to the subject of this article.

\section{REFERENCES}

Bohlman HH. Current concepts review: Treatment of fractures and dislocations of the thoracic and lumbar spine. J Bone Joint Surg [Am] 1985; 67-A :165-9.

Denis $F$. The three column spine and its significance in the classification of acute thoracolumbar spinal injuries. Spine 1983;8:817-31.

Dorr LD, Harvey JP Jr, Nickel VL. Clinical review of the early stability of spine injuries. Spine 1982; 7:545-50.

Faerber EN, Wolpert SM, Scott RM, Belkin SC, Carter BL. Computed tomography of spinal fractures. J Comput Assist Tomogr 1979; 3:657-61.

Gertzbein SD, Offierski C. Complete fracture-dislocation of the thoracic spine without spinal cord injury; a case report. J Bone Joint Surg [Am] 1979; 61-A :449-51.

Holdsworth F. Review article: fractures, dislocations, and fracture-dislocations of the spine. J Bone Joint Surg [Am] 1970; 52-A: 1534-51.

Hunter JC, Fink IJ, Zinreich SJ, Chafetz N, McClendon DR. Threedimensional CT imaging of the lumbar spine. In: Genant HK, ed. Spine update 1987: perspectives for radiologists, orthopaedists and neurosurgeons. San Francisco: Radiology Research and Education Foundation, 1987:237-45.

Jacobs RR. Bilateral fractures of the pedicles through the fourth and fifth lumbar vertebrae with anterior displacement of the vertebral bodies: case report. J Bone Joint Surg [Am] 1977; 59-A :409-10.

Lynch D, McManus F, Ennis JT. Computed tomography in spinal trauma. Clin Radiol 1986; 37:71-6.

Pitman MI, Pitman CA, Greenberg IM. Complete dislocation of the cervical spine without neurological deficit: a case report. $J$ Bone Joint Surg [Am] 1977; 59-A:134-5.

Sasson A, Mozes G. Complete fracture-dislocation of the thoracic spine without neurologic deficit : a case report. Spine 1987; 12:67-70.

Ullich CG, Binet EF, Sanecki MG, Kieffer SA. Quantitative assessment of the lumbar spinal canal by computed tomography. Radiology $1980 ; 134: 137-43$. 